\title{
High-Resolution Imaging of Single-Cell Behaviors in 3D Bacterial Biofilms using Lattice-Light Sheet Microscopy and Deep Learning-Based Image Processing
}

\author{
Ji Zhang ${ }^{1}$, Yibo Wang ${ }^{1}$, Mingxing Zhang ${ }^{1}$, Alecia Achimovich ${ }^{1}$, Jie Wang ${ }^{1}$, Scott Acton ${ }^{1}$ and Andreas \\ Gahlmann $^{2}$
}

${ }^{1}$ University of Virginia, United States, ${ }^{2}$ University of Virginia, CHARLOTTESVILLE, Virginia, United States

Myxococcus xanthus is a highly social bacterium and a model system for coordinated multicellular behaviors, including swarming motility and developmental cell differentiation. Under nutrient rich conditions, $M$. xanthuscells migrate over surfaces as dense 2-dimensional swarms to prey on nearby bacterial colonies. Under nutrient limited conditions, $M$. xanthuspopulations activate a developmental program that induces cells to aggregate into 3dimensional mounds. Some of these mounds develop further into fruiting bodies with heights and diameters of up to 100 micrometers. Both swarming motility and fruiting body development involve pronounced changes in cell morphology. Swarming, rod-shaped M. xanthuscells can bend along their long axis by well over 90 degrees (Figure 1a). During fruiting body development, rod-shaped cells either differentiate into spherical myxospores or into peripheral rods (a persister-like cell-state), or they burst by undergoing developmental cell lysis (Figure 1b). While these morphological transitions have been observed for isolated cells, or in larger populations at single, fixed timepoints, it has not been possible to follow the migration behaviors and developmental fates of hundreds and thousands of cells in a population over time. As such, it remains unclear how different biochemical signals and/or mechanical cues help coordinate cell migration and cell differentiation in 3D space and time.

Conventional fluorescence imaging modalities suffer from phototoxicity and photobleaching limitations that prevent high-resolution, single-cell tracking of motility or developmental progression over hours and days in large 3D bacterial communities, called biofilms. To address this challenge, we adapted minimally-invasive lattice light-sheet microscopy (LLSM) for 3D bacterial biofilm imaging within optically accessible flow cells. LLSM combines state-ofthe-art 3D spatial resolution (300-400 nm) with fast temporal resolution (up to $100 \mathrm{~ms}$ for single-cell imaging) and low photodamage at levels that cannot be matched by confocal microscopy 1,2 . Specifically, LLSM (and light sheet microscopy modalities in general) provide about an order of magnitude lower photobleaching rates compared to confocal microscopy 3,4 . While LLSM enables high-resolution, long-term time-lapse imaging of bacterial biofilms, the spatial resolution is still comparable to the size of single bacterial cells ( $800 \mathrm{~nm}$ in diameter) and, therefore, intercellular spaces remain difficult to resolve when cells are densely packed. An additional complication is that bacteria scatter excitation and emission light, so that the signal-to-background ratio (SBR) in 3D biofilm images degrades rapidly with increasing depth. These properties make it challenging to identify and outline individual cells in 3D biofilms, even with state-of-the-art light sheet-based imaging approaches.

To automatically identify and outline individual cells in 3D biofilm images, we developed Bacterial Cell Morphometry 3D $(B C M 3 D)^{5}$, an open-source, integrated image analysis workflow that currently achieves state-of-the-art performance in 3D biofilm image segmentation. BCM $3 D$ combines deep learning by convolutional neural networks (CNNs) with mathematical image analysis to automatically recognize characteristic morphological features in 3D images of densely packed bacterial biofilms. To avoid the time-investment and inconsistencies associated with human dataset annotation, $B C M 3 D$ pioneers the use of computationally simulated biofilm images to train $3 D$ U-Net ${ }^{6}$ CNNs. In its initial implementation $\frac{5}{5}$, the in silico-trained CNNs of $B C M 3 D$ consistently outperform previous biofilm segmentation approaches $\frac{7-10}{2}$ in terms of cell counting accuracy and cell shape estimation over a wide range of signal-to-background ratios and cell densities. BCM $3 D$ further enables morphometric cell classification in mixedspecies biofilms based on different cell shapes or different fluorescent labeling/staining approaches $\underline{\underline{5}}$.

In recent work, we further expanded the $B C M 3 D$ workflow with complementary CNN-based processing pipelines that transform the raw 3D fluorescence images into strategically altered images that are more amenable to conventional mathematical image processing, such as watershed segmentation. Initial results show similar, and in some cases superior, segmentation performance compared to the initial $B C M 3 D$ workflow. Importantly, the new $B C M 3 D$ pipelines do not require image deconvolution as a pre-processing step and are able to recognize a larger variety of bacterial cell shapes, including straight rods, spherical cells, as well as regular and irregularly bent cells. 
By integrating versatile and complimentary approaches for 3D single cell segmentation, we obtain accurate and independently validated single-cell segmentation results. The ability to acquire and automatically analyze 3D images and 4D movies of hundreds and thousands of $M$. xanthuscells over orders of magnitude in length- and timescales is a critical prerequisite for measuring the diversity of the phenotypic and developmental trajectories of individual cells during multicellular swarming and fruiting body formation. Understanding the growth and function of bacterial biofilms in terms of the behavioral phenotypes of individual cells will help further our understanding of how bacteria in general utilize biochemical and mechanical signals to coordinate their cellular behaviors in multicellular biofilms 11,12 .
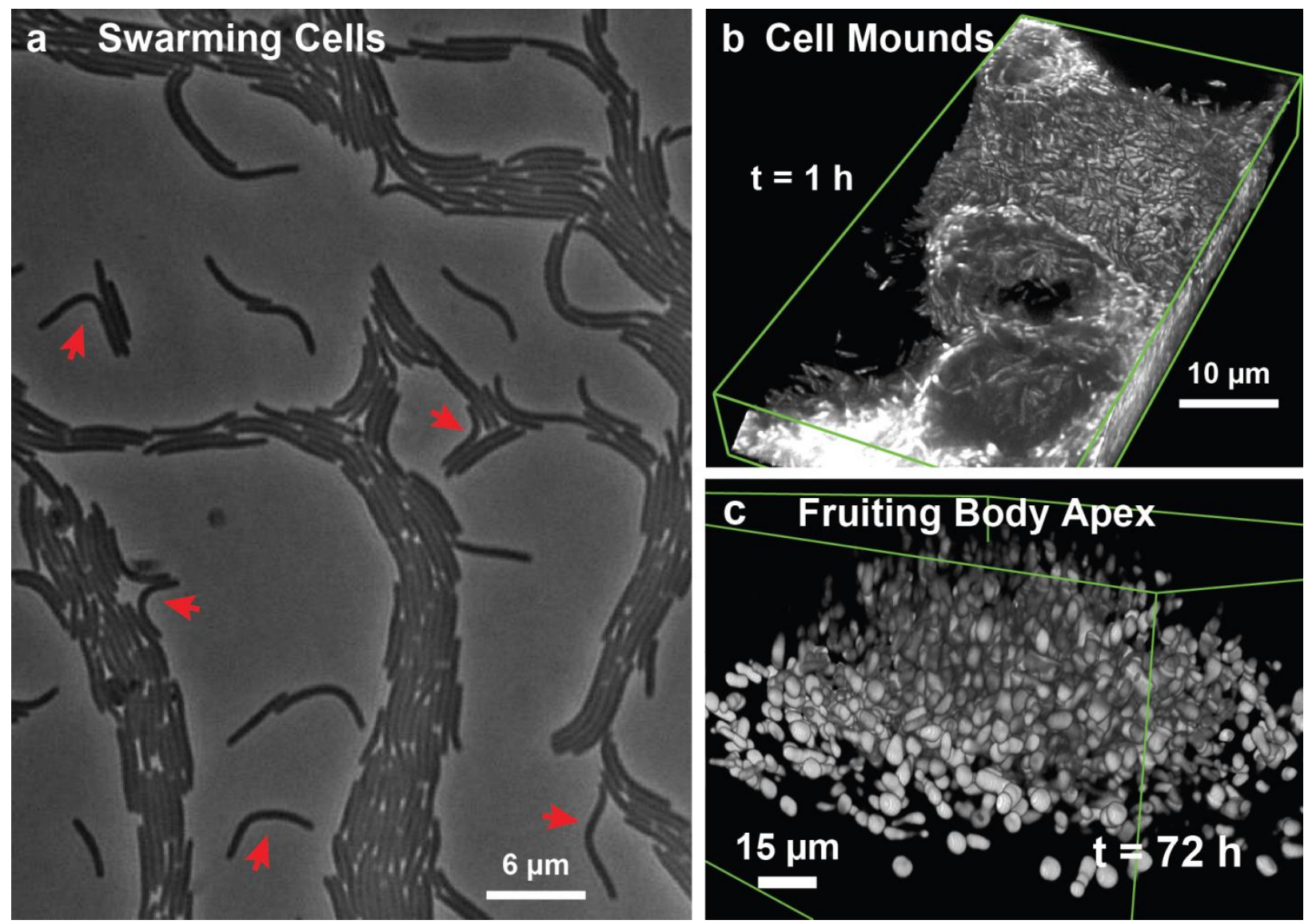

Figure 1. Figure 1. Dynamic cell bending and morphological cell differentiation in multicellular $M$. xanthus communities. (a) Swarming M. xanthus cells on a flat surface. Cells generally adopt a straight-rod shape, but cells can also bend along their long axis (indicated by red arrows). (b) Rod-shaped M. xanthus cells aggregate into 3D mounds after being placed in starvation buffer. Some of these mounds later differentiate into spore-filled fruiting bodies. (c) 3D image of the apex of a mature M. xanthus fruiting body mostly consisting spherical myxospores.

References

1. Chen, B.C., et al. Lattice light-sheet microscopy: imaging molecules to embryos at high spatiotemporal resolution. Science 346, 1257998 (2014).

2. Li, D., et al. Extended-resolution structured illumination imaging of endocytic and cytoskeletal dynamics. Science 349, aab3500 (2015).

3. Yang, B., et al. Epi-illumination SPIM for volumetric imaging with high spatial-temporal resolution. Nat Methods 16, 501-504 (2019).

4. Voleti, V., et al. Real-time volumetric microscopy of in vivo dynamics and large-scale samples with SCAPE 2.0. Nat Methods 16, 1054-1062 (2019). 
5. Zhang, M., et al. Non-invasive single-cell morphometry in living bacterial biofilms. Nature Communications 11, 6151 (2020).

6. Çiçek, Ö., Abdulkadir, A., Lienkamp, S.S., Brox, T. \&Ronneberger, O. 3D U-Net: Learning Dense Volumetric Segmentation from Sparse Annotation. 424-432 (Springer International Publishing, Cham, 2016).

7. Stringer, C., Wang, T., Michaelos, M. \&Pachitariu, M. Cellpose: a generalist algorithm for cellular segmentation. Nat Methods 18, 100-106 (2021).

8. Yan, J., Sharo, A.G., Stone, H.A., Wingreen, N.S. \&Bassler, B.L. Vibrio cholerae biofilm growth program and architecture revealed by single-cell live imaging. Proc Natl Acad Sci U S A 113, E53375343 (2016).

9. Reyer, M.A., McLean, E.L., Chennakesavalu, S. \& Fei, J. An Automated Image Analysis Method for Segmenting Fluorescent Bacteria in Three Dimensions. Biochemistry 57, 209-215 (2018).

10. Hartmann, R., et al. Emergence of three-dimensional order and structure in growing biofilms. Nature Physics 15, 251-256 (2019).

11. Kroos, L. Highly Signal-Responsive Gene Regulatory Network Governing Myxococcus Development. Trends Genet 33, 3-15 (2017).

12. Lee, C.K., et al. Multigenerational memory and adaptive adhesion in early bacterial biofilm communities. Proc Natl Acad Sci U S A (2018). 